tenmonaten $\mathrm{zu}$ kalkulieren ist. Insgesamt beträgt die Häufigkeit von schwerwiegenden kardiovaskulären Nebenwirkungen etwa 0,2 bis 0,4 Fälle pro 10 Millionen eingenommener Tabletten.

Das Nebenwirkungsprofil von Teldane ${ }^{\circledR}$ ist damit sehr gering und liegt in der Größenordnung anderer moderner Antihistaminika.

\section{Anmerkungen zur Sendung}

Zu den in der „Report"-Sendung vom 2. Juni 1997 vorgestellten Einzelfällen kann Hoechst Marion Roussel keine Stellung nehmen, da Nebenwirkungen anonym gemeldet (nur Initialien) werden. Alle eingehenden Fälle werden wissenschaftlich bewertet und an das BfArM weitergegeben.

Hoechst Marion Roussel und das Bundesinstitut für Arzneimittel und Medizinprodukte arbeiten gemeinsam, gemäß dem Artikel 12, daran, das Sicherheitsprofil von Teldane ${ }^{\circledR}$ für den Patienten weiterzuentwickeln.

Dem Unternehmen sind keine $\mathrm{Ne}$ benwirkungen infolge einer Interaktion zwischen Terfenadin und Grapefruitsaft bekannt. Aufgrund pharmakokinetischer Untersuchungen kann vermutet werden, daß es bei gleichzeitiger Einnahme von Terfenadin und Grapefruitsaft $\mathrm{zu}$ einer Erhöhung des TerfenadinWirkspiegels kommen kann. Im Beipackzettel wird seit Januar 1997 darauf hingewiesen, daß die Einnahme von Teldane ${ }^{\circledR}$ nicht gleichzeitig mit größeren Mengen Grapefruitsaft erfolgen soll.

Dr. Dieter Görte ist nicht Pressesprecher der Hoechst AG, wie in der Sendung behauptet, sondern medizinischer Direktor von Hoechst Marion Roussel Deutschland $\mathrm{GmbH}$.

Hoechst Marion Roussel steht zu seiner Verantwortung als Hersteller von Teldane $^{\circledR}$. Zu dieser Verantwortung gehört es, daß das Unternehmen konstant das Nutzen-Risiko-Verhältnis des Präparates bewertet und an Ärzte, Apotheker und Patienten weitergibt.

Quelle: Presse-Information der Hoechst Marion Roussel Deutschland $\mathrm{GmbH}$

\title{
Atemwegsprobleme nach dem ersten Lebensjahr erhöhen Asthmarisiko
}

Infektionen der unteren Atemwege und respiratorische Symptome wie Husten und Giemen sind bei Säuglingen und Kleinkindern relativ häufig. Treten derartige Beschwerden auch noch nach dem ersten Lebensjahr und ohne begleitende Erkältung auf, kann dies der erste Hinweis auf ein beginnendes Asthma sein.

$\mathrm{E}$ ne amerikanische Arbeitsgruppe aus Tucson/Arizona beobachtete in einer repräsentativen Longitudinalstudie 786 Kinder über einen Zeitraum von 3 bis 11 Jahren. Bei Aufnahme in die Studie hatte keines der Kinder das 5. Lebensjahr überschritten, drei Viertel der Probanden waren jünger als 2 Jahre alt.

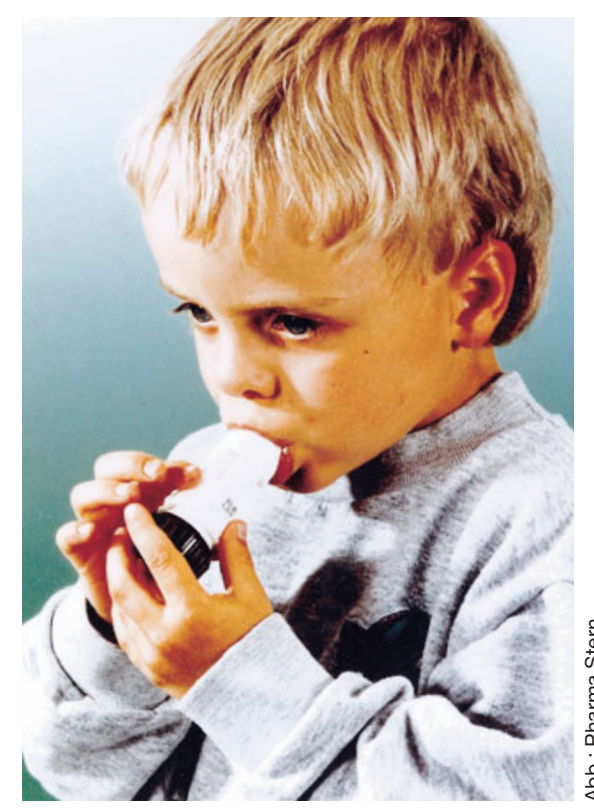

Kindliches Asthma: Erstes Warnsymptom können Kurzatmigkeit und vermehrter Husten nach dem ersten Lebensjahr sein.
Der Gesundheitszustand der Kinder wurde über Fragebogen ermittelt, die alle ein bis zwei Jahre an die Eltern geschickt wurden.

\section{Ergebnisse}

Im ersten Lebensjahr auftretende respiratorische Symptome, von Erkältungen über Husten bis zu Giemen ohne Erkältung, hatten einzeln betrachtet keinen signifikanten Effekt auf die spätere Asthmainzidenz. Nur wenn alle drei Symptome zusammenkamen, war das Asthmarisiko gegenüber Kindern ohne eines der Symptome um den Faktor 3,2 erhöht.

Anders sah es bei Kindern aus, bei denen Husten, Giemen ohne Erkältung oder Kurzatmigkeit zwischen dem ersten und zweiten Lebensjahr auftraten. Hier war das Risiko eines späteren Asthma bronchiale signifikant erhöht (Odds Ratio 2,1).

Noch ausgeprägter war dieser $\mathrm{Zu}$ sammenhang bei den 3- bis 4jährigen. Wurden in diesem Alter chronischer Husten, Giemen ohne Erkältung oder Kurzatmigkeit angegeben, war das Asthmarisiko um den Faktor 8,5 erhöht. Anders ausgedrückt: $30 \%$ dieser Kinder entwickelten später ein Asthma bronchiale.

Zusammenfassend können damit respiratorische Symptome wie $\mathrm{Hu}-$ sten, Kurzatmigkeit und nicht auf Erkältungen zurückzuführendes Giemen, die bei Kindern nach dem 1 . Lebensjahr auftreten, als Risikofaktoren für Asthma angesehen werden. Daß diese Assoziation mit steigendem Alter an Bedeutung gewinnt, könnte nach Ansicht der Autoren daran liegen, daß respiratorische Symptome im ersten Lebensjahr eher auf unkomplizierte virale Infektionen zurückzuführen sind, während spätere Beschwerden bereits eine beeinträchtigte Funktion der unteren Atemwege anzeigen.

$(z o)$

Quelle: Dodge R, Martinez FD, Cline MG et al. Early childhood respiratory symptoms and the subsequent diagnosis of asthma. J Allergy Clin Immunol 1996; 98:48-54. 